\title{
Dynamic Contact Characteristics of Elastic Composite Cylindrical Roller Bearing
}

\author{
Jianghong Yu, Ran Zhang, Wen Yang and Qishui Yao*
}

Hunan University of Technology, Zhuzhou, 412007, China

\begin{abstract}
Elastic composite cylindrical roller bearing is a kind of new bearing. In view of its structural particularity, explicit dynamics finite element model of elastic composite cylindrical roller bearing is established by utilizing ABAQUS/EXPLICIT. Dynamic responses of elastic composite cylindrical roller bearing are analyzed and response analysis is compared under different radial loads and rotation speeds. Dynamic responses of elastic composite cylindrical roller bearing are analyzed and response analysis is compared under different radial loads and rotation speeds. Results show that rolling and holder lag in rotation is as being compared to inner ring. The motion processes of all the holder, inner ring and roller have certain periodicity. Fluctuation amplitude of inner ring displacement increases with load. Response increases with rotation speed when amplification decreases. Analysis results can offer beneficial reference for further research on dynamic characteristics of elastic composite cylindrical roller bearing.
\end{abstract}

Keywords: Elastic composite cylindrical roller bearing, explicit dynamics, finite element, radial load, rotation speed.

\section{INTRODUCTION}

Rolling bearing is a kind of widely used parts in mechanical equipment in the modern industry [1]. Its performance plays an important role in the entire mechanical system. It is necessary to research dynamic performance of rolling bearing to guarantee the reliability of the entire system. Domestic and foreign scholars have researched dynamic performance of rolling bearing. Jones first proposes collar control theory of high-speed ball bearing and establishes quasi static analysis method [2]. Harris further improves the quasi static analysis method of rolling bearing [3] by considering the influence to EHL effect and inertia force on the basis of Jonse's research. Gupta establishes 6 degrees of freedom of a dynamic model of rolling bead (ball bearing), analyzes bearing motion relationship and proposes analysis a model of rolling bearing [4] under any simulation operation condition. Hu Xuan from Nanjing University of Aeronautics and Astronautics establishes a non-linear dynamic analysis mathematical model of high-speed cylindrical roller bearing and calculates dynamic characteristics of cylindrical roller intermediate bearing and compares calculated roller slippage with test results [5].

Documents [6-8] propose a new rolling bearing, the elastic composite cylindrical roller bearing, to adapt to requirements for high rotation speed and high precision. This kind of bearing is to embed PTFE materials into rolling of hollow cylindrical roller bearing, so as to improve stress status of inner wall of rolling, reduce bending fatigue stress of inner wall and prolong safety service life of the bearing. Due to the structural complexity of the elastic composite cylindrical roller bearing, it is necessary to research integral

*Address correspondence to this author at the Hunan University of Technology, Zhuzhou, 412007, China; Tel: +86-731-22183978;

E-mail: yaoqishui@126.com elastic deformation of the elastic composite rolling during motion process. Finite element simulation is widely used in bearing dynamic research as an important tool to analyze contact problem of some complex structures. Wei Yangang analyzes edge effect of hollow and solid cylindrical roller bearings during use, and proposes a new deep cavity hollow roller bearing [9] by using ANSYS software. Xu Hongyi establishes a multi-body dynamic contact finite element model of roller bearing, and calculates stress conditions [10] of low-speed bearings of different deformation constitutive relationship material model under two operating conditions by selecting two different plastic deformation models: bilinear isotropic model (BISO) and bilinear kinematic hardening model.

This paper mainly researches the rolling stress variation and deformation in dynamic process by using the elastic composite cylindrical roller bearing as an analysis target. The solid model of the elastic composite cylindrical roller bearing is established in Pro/E software, and ABAQUS is imported for finite element analysis. Stress variation, displacement, speed and acceleration during rotation process are lucubrated, and comparisons are made on stress and displacement variation of elastic composite cylindrical roller bearing of different radial loads and rotation speeds. Useful references can be provided in the results for future research onthe elastic composite cylindrical roller bearing.

\section{BASIC THEORY OF EXPLICIT DYNAMICS}

Implicit integration method is employed inthe static analysis on finite element. The method has the advantages of long integration step and fast calculation; and disadvantage of large space consumption for quasi rigidity matrix solution in calculation. Step wise solution is iterated and reduced continuously and rigidity matrix of high calculation cost is reconstructed when a highly nonlinear problem is solved in 
less time increment then required as compared to the implicit integration method. Iteration and convergence criterion are not needed, nor do the integral tangent rigidity matrix. Calculation time can be reduced by suitable mass scaling. Therefore, the explicit integration method can save a lot of time as compared to the implicit integration method. It logically follows that the former is adopted to establish finite element analysis model.

ABAQUS/Explicit model is mainly used for high-speed collision, complex contact, material invalidation and degeneration simulation analysis [11]. Calculation principle is to carry out explicit integration of motion equation by adopting central difference method, and to calculate dynamic conditions of next increment step based on dynamic conditions of one increment step. Dynamic balance equation of bearing system will be calculated [12] as follows at the moment of increment step:

$M \ddot{u}+C \dot{u}+K u=P$

where, M: Quality matrix;

$\mathrm{C}$ : Damping matrix;

$\mathrm{K}$ : Rigidity matrix;

$\ddot{u}$ : Acceleration vector of system node;

$\dot{u}$ : Speed vector of system node;

$u$ : Displacement vector of system node;

P: Load vector of the node.

Acceleration calculation formula is as follows at the moment of increment step:

$\ddot{u}_{(t)}=(M)^{-1}(P-C \dot{u}-K u)_{(t)}$

Central difference method is employed to integrate acceleration which is assumed as a constant when speed variation is calculated. The middle point speed of current increment step is determined by the combination of speed variation and middle point speed of previous increment step as follows:

$\dot{u}_{\left(t+\frac{\Delta t}{2}\right)}=\dot{u}_{\left(t-\frac{\Delta t}{2}\right)}+\frac{\left(\Delta t_{t+\Delta t}+\Delta t_{t}\right)}{2} \ddot{u}_{(t)}$

where, $\Delta t$ : Calculated time step.

Displacement is determined when increment step ends by the combination of speed-time integral and displacement when increment step begins as follows:

$u_{(t+\Delta t)}=u_{\Delta t}+\Delta t_{(t+\Delta t)} \dot{u}_{\left(t+\frac{\Delta t}{2}\right)}$

Time step $\Delta t$ depends on estimated units one by one; Limit time step of unit is as follows:

$\Delta t_{s}=\frac{L^{e}}{C_{d}}$

where, $\mathrm{L}^{\mathrm{e}}$ is: Unit length; $\mathrm{C}_{\mathrm{d}}$ is : Wave speed of materials

$C_{d}=\sqrt{\frac{E}{\rho}}$ where, $\mathrm{E}$ is Elastic modulus of materials; $\rho$ is: Material density.

\section{FINITE ELEMENT MODEL}

\subsection{Geometric Parameters of Elastic Composite Cylindrical Roller Bearing}

Geometric parameters of the elastic composite cylindrical roller bearing are analyzed in this paper by selecting NU318E bearing as an analysis target according to the national standards GB/T4661-2002 and GB/T283-2007. Related parameters are shown in Table 1:

Table 1. Physical dimension parameters of NU318E bearing.

\begin{tabular}{|c|c|}
\hline Parameter & Value \\
\hline \hline Outer diameter of bearing/mm & 190 \\
\hline Inner diameter of bearing/mm & 90 \\
\hline Width of Bearing/mm & 43 \\
\hline Length of Rolling element $/ \mathrm{mm}$ & 30 \\
\hline Diameter of Rolling element $/ \mathrm{mm}$ & 28 \\
\hline Number of Rolling elements & 13 \\
\hline Filling degree & $55 \%$ \\
\hline
\end{tabular}

The 3D model is established and ABAQUS finite element software is imported according to the geometric parameters as shown in Table $\mathbf{1}$.

\subsection{Establishment of Finite Element Model}

\subsubsection{Material Parameter}

The deformation of inner or outer bearing ring in actual operating conditions is very small and is not the focal point of this paper. Inner or outer bearing ring is defined as rigid body to increase the efficiency and reduce the calculation time. Outer ring of rolling body, inner or outer bearing ring and holder which are made of bearing steel are selected. Density is $7,800 \mathrm{Kg} / \mathrm{m}^{3}$ and elastic modulus is $2.06 \times 10^{5} \mathrm{MPa}$. Poisson ratio is 0.3 ; Inner filling is PTFE with a density of $2,200 \mathrm{Kg} / \mathrm{m}^{3}$; Elastic modulus is $280 \mathrm{MPa}$ and Poisson ratio is 0.4 .

\subsubsection{Division of Unit Type and Grid and Setting of Analysis Steps}

Grid is divided by linear reduction integral hexahedron unit of unit type C3D8R for all units of bearing to be analyzed [13, 14]. Reasonable grid division not only guarantees the accuracy of calculation results, but also effectively increase the calculation efficiency [15]. Therefore, grid division is the analysis emphasis of the finite element. The main analysis target is the contact problem of rolling and inner and outer rings. Rolling is the focus while the holder and the inner and outer rings are not, because of little influence on calculation results. Therefore, the grid division of the outer ring of rolling and the inner filling is more accurate than the division of the holder and the inner 
and outer rings. The impact of different densities on the calculation results by the finite element analysis, as shown in Fig. (1), indicates that the grid division of 11164505 nodes and 5532672 units is reasonable.

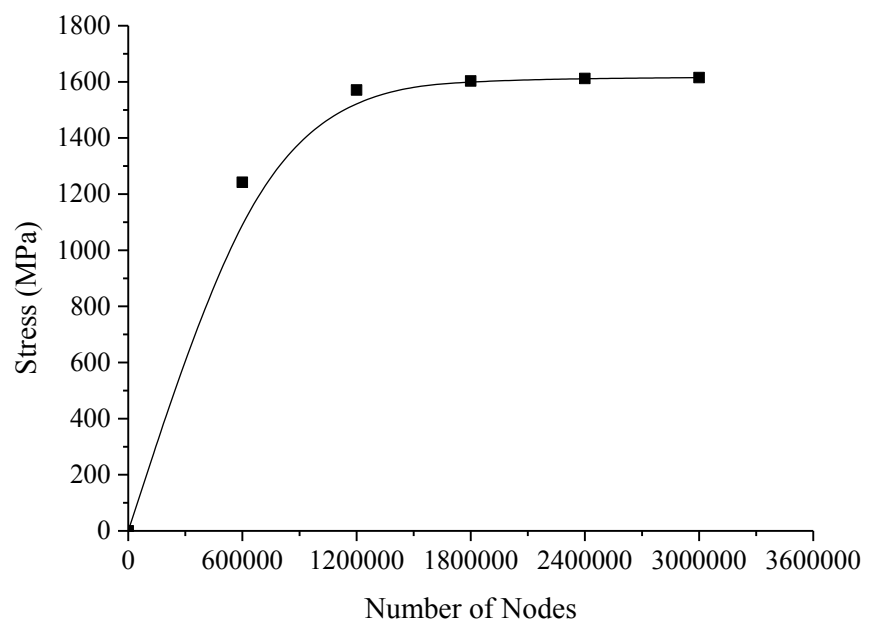

Fig. (1). The relationship between stress value and the number of node.

Three steps are set in the analysis. In the first step, the gravity is exerted. In the second step, the radial load is exerted. In the third step, the rotation speed for inner ring is exerted. Field variable output of radial displacement of the inner ring and the stress variation of contact point are set.

\subsubsection{Contact, Border Conditions and Load}

Two reference points RP1 and RP2 are created in the model. RP1 is at the middle of the inner ring axle and RP2 is atthe upper part of the outer ring. As shown in Fig. (2), RP1 and the inner surface of the inner bearing ring are coupled and then rigid restriction on the inner ring is exerted; RP2 and the outer surface of outer bearing ring are coupled and then rigid restriction on the outer ring is exerted; the outer ring of the rolling and the inner filling are tied and restricted. Surface-to-surface contact is used and totally 39 contact pairs between the outer surface of the rolling outer surface of the inner ring, the inner surface of the outer ring and the holder respectively with a friction coefficient of 0.15 is established.

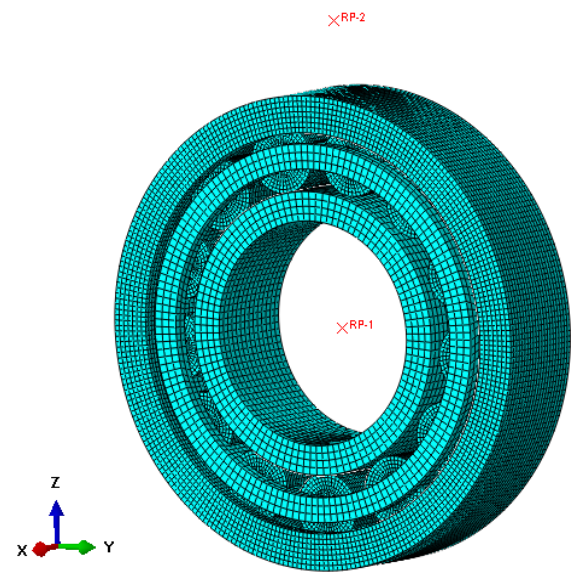

Fig. (2). Finite element model of elastic composite cylindrical roller bearing.
Border conditions are exerted by selecting the border restriction scheme of the rotary inner ring and fixed the outer ring to make the analysis more practicable. Degree of freedom of translation toward $X$ axle and rotation degree of freedom toward $\mathrm{Y}$ and $\mathrm{Z}$ axles of the inner ring are restricted in the initial step. Restraint of totally 6 degrees of the freedom for the outer ring (on reference point RP1) is exerted but no restriction for the holder is exerted. Gravity load toward negative direction of $\mathrm{Z}$ axle on $\mathrm{Z}$ axle is exerted in the first step. Radial load toward negative direction of $Z$ axle on the inner ring (on reference point RP2) is exerted in the second step. Bearing should also bear effect of centrifugal force caused by its rotation during the operation process. Clockwise rotation border conditions around $\mathrm{X}$ axle on the inner ring (on reference point RP2) is exerted in the third step.

\section{SIMULATION RESULT ANALYSIS AND DISCUSSION}

Dynamic response analysis is carried out during the operation process of the elastic composite cylindrical roller bearing, and the influence of different radial loads or the rotation speeds on dynamic response is compared by using the above finite element analysis model. Center point RP1 of the inner ring and the initial position of rolling unit which bears the maximum load are selected as research target to facilitate the observation and the analysis.

\subsection{Radial Displacement of Inner Ring and Stress Analysis of Contact Point}

The finite element model is established under the radial load of $100 \mathrm{KN}$, the rotation speed of $2,000 \mathrm{r} / \mathrm{min}$ and the filling degree of $55 \%$ to analyze the radial displacement response of the inner ring and stress response of the initial position of 1 rolling point toward the radial load direction. RP1 and contact center of the initial position on the rolling toward the radial load direction and the inner ring are set on respectively. Displacement response curve of the inner bearing ring within $0.2 \mathrm{~s}$ is shown in Fig. (3). The stress response curve of rolling and the inner ring contact point is shown in Fig. (4).

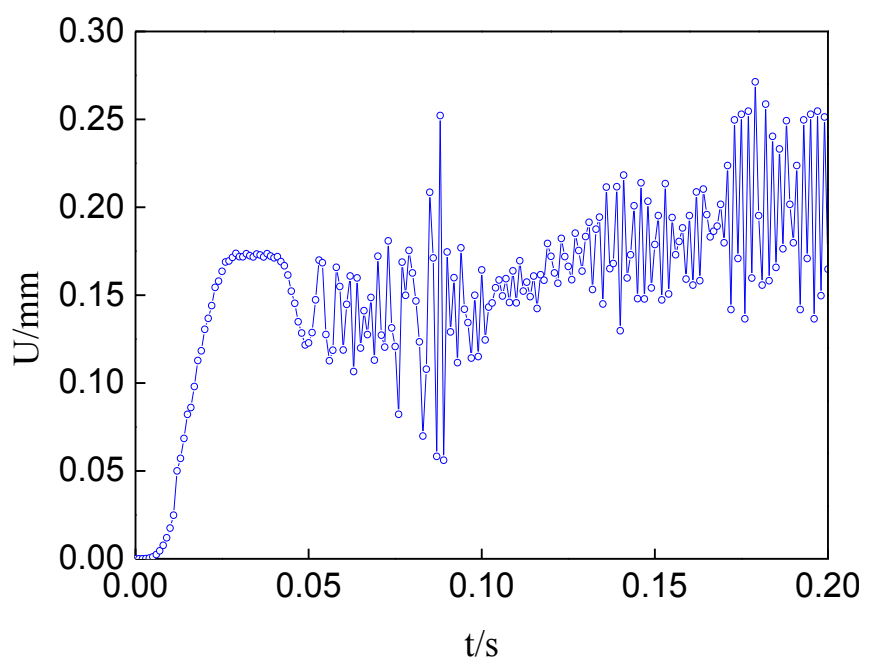

Fig. (3). Displacement response diagram of inner ring of elastic composite cylindrical roller bearing. 


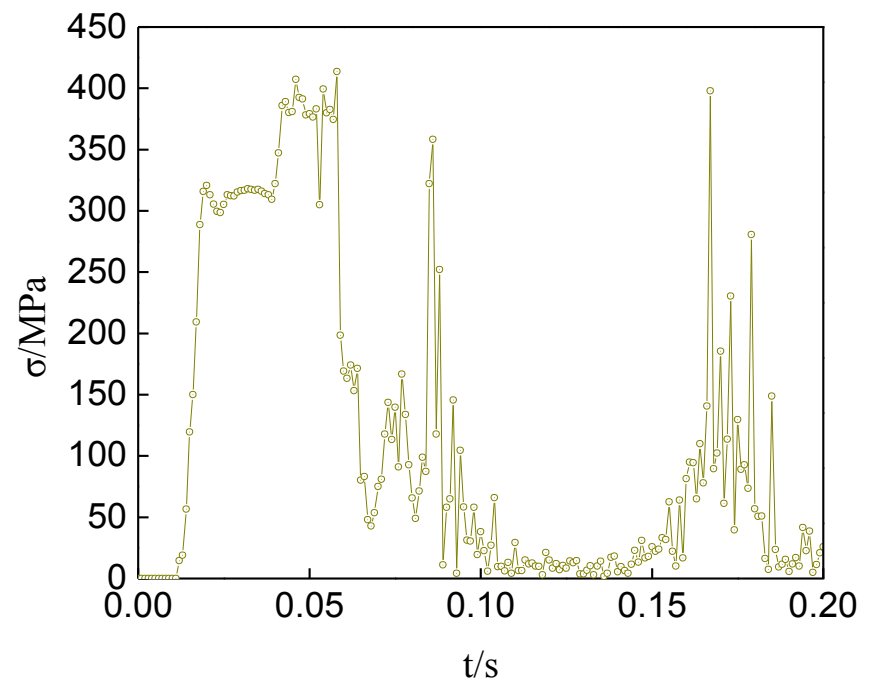

Fig. (4). Stress variation diagram of elastic composite cylindrical roller bearing rolling.

From Fig. (3), the displacement of the inner bearing ring is almost always 0 when the gravity load is added from 0 to $0.01 \mathrm{~s}$, so the gravity influence on the bearing analysis can be neglected. Displacement of the inner bearing ring constantly increases from $0.01 \mathrm{~s}$ to $0.03 \mathrm{~s}$ because the rolling will have contact deformation when exerting the radial load. Radial displacement of the inner ring is about $0.08 \mathrm{~mm}$ after exerting load. Rotation speed is exerted on the inner ring from $0.03 \mathrm{~s}$, while the radial displacement varies obviously till $0.045 \mathrm{~s}$. As it is shown that rolling lags in rotation as compared to the inner ring. Displacement of the inner ring decreases quickly from $0.045 \mathrm{~s}$ because the rolling distribution is even pressure status at $0.045 \mathrm{~s}$. It changes to odd the pressure status with the rotation of the inner ring, so displacement decreases quickly at $0.045 \mathrm{~s}$. Then the displacement varies continuously with the circulated and repeated pressure. The displacement varies greatly at about $0.09 \mathrm{~s}$ then stabilizes gradually. The displacement reduces to $0.2 \mathrm{~mm}$.

From Fig. (4), the rolling has almost no stress when exerting the gravity load from 0 to $0.01 \mathrm{~s}$; The rolling node stress increases gradually with the radial load from $0.01 \mathrm{~s}$ to $0.03 \mathrm{~s}$; the stress decreases obviously until after $0.045 \mathrm{~s}$ because the rolling rotation lags in rotation as compared to the inner ring after $0.03 \mathrm{~s}$, and this node leaves contact position from $0.045 \mathrm{~s}$ and the stress decreases quickly. The rolling will contact the inner and outer rings periodically besides, the stress increases when contacting. In the meanwhile, it collides with the holder and causes the node stress fluctuation. The rolling contacts with the inner ring and causes the peak stress at about $0.09 \mathrm{~s}$ and $0.17 \mathrm{~s}$.

\subsection{Node Displacement Response Contrast Analysis of Different Parts}

The finite element model is established under the radial load of $100 \mathrm{KN}$, the rotation speed of $2,000 \mathrm{r} / \mathrm{min}$ and the filling degree of $55 \%$. The displacement response of the bearing holder, the inner ring and the rolling nodes toward $\mathrm{Z}$ direction are analyzed. One node is selected on the outer surface of the inner ring, the rolling contact center, the contact center of the rolling and the inner ring as well as the holder, respectively to analyze the displacement response of these three nodes. As shown in Fig. (5).

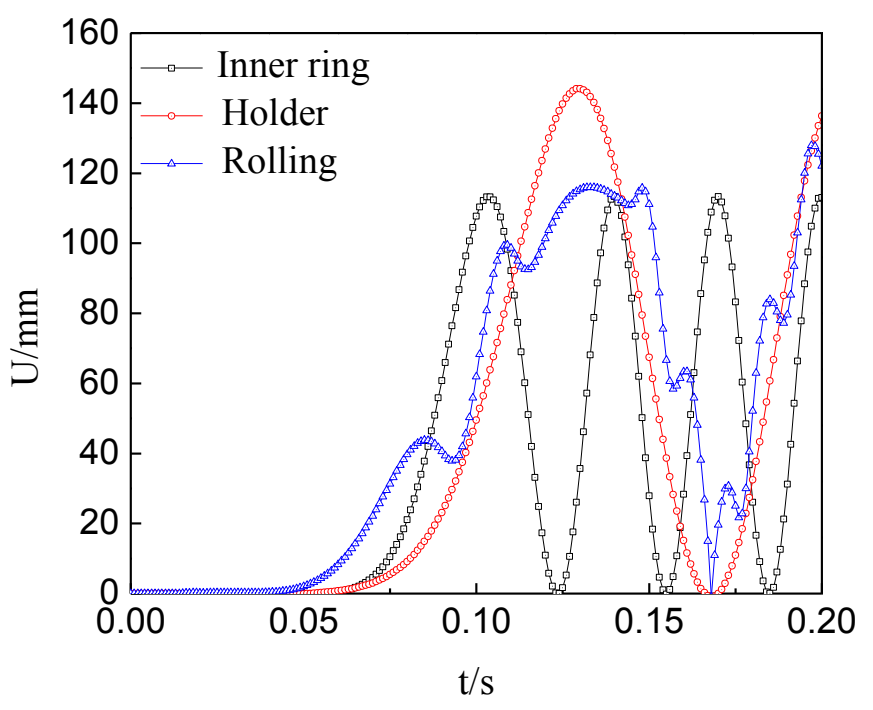

Fig. (5). Displacement response diagram of holder, inner ring and rolling node toward $\mathrm{Z}$ direction.

The displacement response of the holder, the inner ring and the rolling nodes toward $\mathrm{Z}$ direction can be found in Fig. (5). Certain periodicity during the motion process is presented. The displacement response curve of the inner ring and the holder nodes is a sine curve while the displacement response of the rolling node is irregular because the rolling will collide with the holder during the rotation process. When the node of the inner ring and rolling completes one circle, the movement displacement is $110 \mathrm{~mm}$; when the node of the holder completes one circle, the movement displacement is $140 \mathrm{~mm}$. The cycle of the inner ring is about $0.03 \mathrm{~s}$. Cycles of the holder and the rolling are approximately equal, namely, about $0.075 \mathrm{~s}$. Of which: the inner ring rotates by 1 cycle at $0.125 \mathrm{~s}$ while the holder and the rolling rotate by 1 cycle about $0.17 \mathrm{~s}$.

\subsection{Displacement Contrast Analysis of Inner Bearing Ring under Effect of Different Radial Loads}

Rotation speed of 1,000r/min and filling degree of 55\% are selected. The radial load of $1 \mathrm{KN}, 10 \mathrm{KN}$ and $100 \mathrm{KN}$ are set, respectively. The above finite element analysis model is established, the result is shown in Fig. (6).

From the above figures, the initial displacement of the bearing increases with radial load. The inner bearing ring has the displacement fluctuation with the rotation. Besides, the displacement fluctuates greatly after $0.1 \mathrm{~s}$. Of which: there is the maximum fluctuation amplitude under $\mathrm{F}=100 \mathrm{KN}$, the secondary value under $\mathrm{F}=10 \mathrm{KN}$ and the minimum value under $\mathrm{F}=1 \mathrm{KN}$. The rolling load deformation increases with load so the inner ring is always under a large displacement variation. Therefore, the displacement fluctuates greatly and reaches a relatively stable status after $0.15 \mathrm{~s}$. Under steady state, the convergence value of the initial displacement of the bearing increases with the load. 


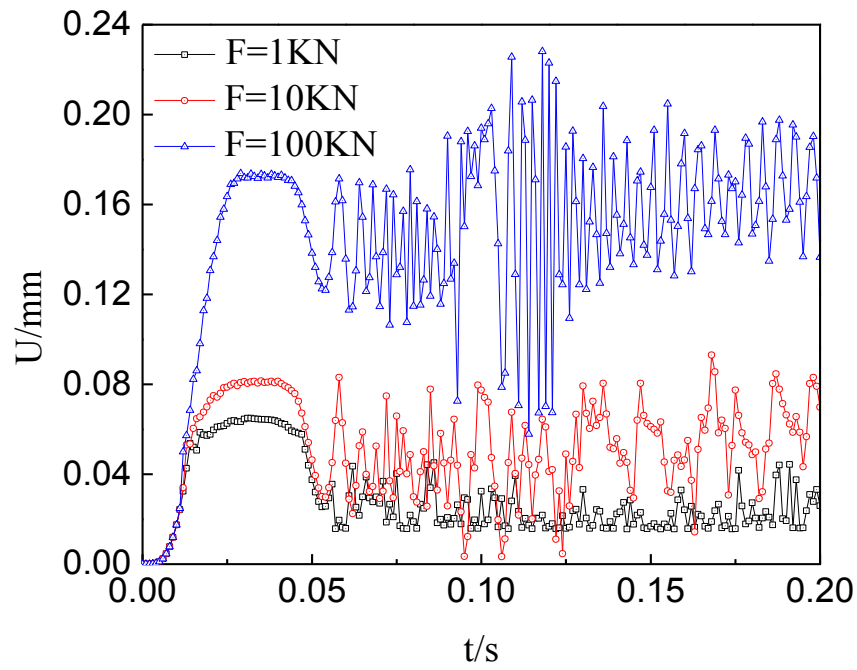

Fig. (6). Displacement response comparison of inner ring under different loads and rotation speed of $1,000 \mathrm{r} / \mathrm{min}$.

\subsection{Displacement Comparison Analysis of Inner Bearing Ring under the Effect of Different Rotation Speeds}

Filling degree of $55 \%$ and radial load of $100 \mathrm{KN}$ are selected. Three finite element analysis models are established under the rotation speed of $1,000 \mathrm{r} / \mathrm{min}$, $2,000 \mathrm{r} / \mathrm{min}$ and 3,000 $/ \mathrm{min}$, respectively. The displacement response of inner ring is shown in Fig. (7).

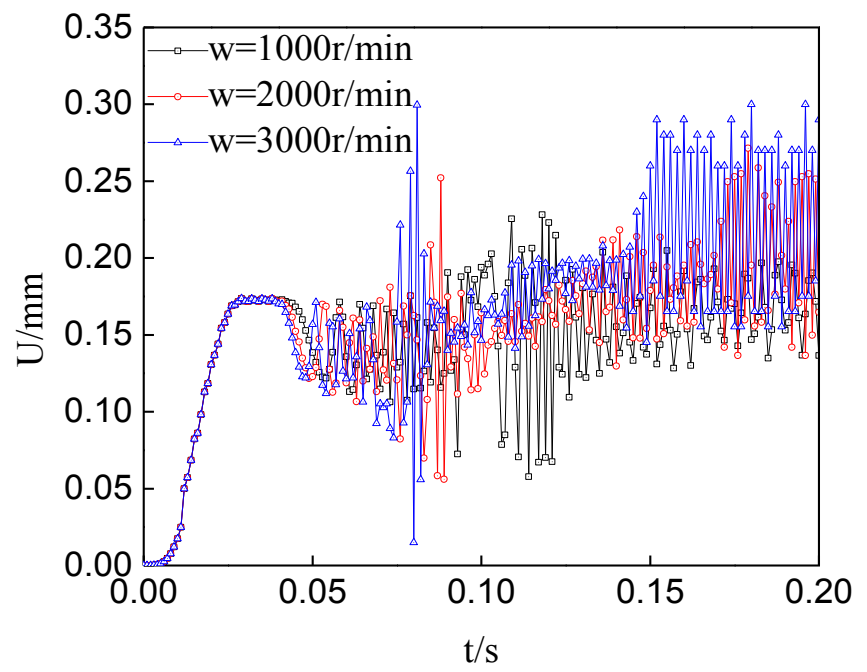

Fig. (7). Displacement response comparison of inner ring under $\mathrm{F}=100 \mathrm{KN}$ and different rotation speeds.

The above figures prove that the displacement of the inner ring is equal under different rotation speeds when exerting the radial load. The quickest response exists under $\mathrm{W}=3000 \mathrm{r} / \mathrm{min}$ after exerting the rotation on the inner ring, the secondary value under $\mathrm{w}=2000 \mathrm{r} / \mathrm{min}$ and the lowest under $\mathrm{w}=1000 \mathrm{r} / \mathrm{min}$. The inner ring displacement fluctuates greatly with the increased rotation speed. The displacement fluctuation increases with the rotation speed then it stabilizes relatively after the fluctuation. The average displacement of the inner ring also increases with the rotation speed after the stabilization because the centrifugal force, as an additional force, is in proportion to square the rotation speed. The centrifugal force of the bearing increases with the rotation speed. The rolling deformation also increases with the force which exerts the rolling.

\section{CONCLUSION}

1) The Rolling and holder lag in rotation as compared to the inner ring. The displacement of the inner ring fluctuates greatly then stabilizes gradually with circulated and repeated odd and even pressure after exerting the rotation speed on the inner ring. Besides, the gravity has almost no effect on the displacement of the inner ring and on the stress of the rolling contact point. The stress always varies because the rolling collides with the inner ring, the outer ring and the holder continuously. Besides, the maximum stress exists when colliding with the inner ring.

2) The motion processes of the holder, the inner ring and the rolling have a certain periodicity. The holder and the rolling rotation cycles are approximately equal during the motion process. Besides, they exceed the inner ring.

3) The initial displacement of the bearing increases with the radial load. The displacement fluctuation increases with load, so the inner ring is always under a large displacement load. Therefore, the inner displacement of the bearing increases.

4) The response increases with the rotation speed after exerting the rotation on the inner ring while the growth rate decreases. The inner ring displacement fluctuates greatly with the increased rotation speed. The displacement fluctuation increases with the rotation speed then it stabilizes relatively after the fluctuation. The average displacement of the inner ring also increases with the rotation speed after stabilization. Under the steady state, the convergence value of the inner displacement increases with the rotation speed.

\section{CONFLICT OF INTEREST}

The authors confirm that this article content has no conflict of interest.

\section{ACKNOWLEDGEMENTS}

This Project (51175168) is supported by National Natural Science Foundation of China. Key Projects (2014GK4014) are supported by Science and Technology Project of Hunan Province.

\section{REFERENCES}

[1] Z. Huang, and Y. Xie, "Fault diagnosis of roller bearing with inner and external fault based on Hilbert transformation", Journal of Central South University: Science and Technology, vol. 42, pp. 1992-1996, 2011.

[2] B. A. Jones, "A general theory for elastically constrained ball and radial roller bearings under arbitrary load and speed conditions", Journal of Basic Engineering, vol. 82, pp. 309- 320, 1960.

[3] A. T. Harris, M. Mindel, "Rolling element bearing dynamics", Wear, vol. 23, pp. 311-317, 1973. 
[4] K. P. Gupta, "Dynamic of rolling-element bearings part I: cylindrical roller bearing analysis", ASME Journal of Lubrication Technology, vol. 101, pp. 293-304, 1979.

[5] X. Hu, G. Luo, and D. Gao, "Quasi-static analysis of cylindrical roller intershaft bearing", Journal of Aerospace Power, vol. 21, pp. 1069-1074, 2006.

[6] Q. Yao, W. Yang, D. Yu, and J. Yu, "Bending stress of rolling element inelastic composite cylindrical roller bearing", Journal of Central South University, vol. 20, pp. 3437-3444, 2013.

[7] Q. Yao, W. Yang, D. Yu, J. Yu, and R. Zhang, "Research on structure design of elastic composite cylindrical roller bearing", China Mechanical Engineering, vol. 23, pp. 2899-2902, 2012.

[8] Q. Yao, W. Yang, D. Yu, J. Yu, and R. Zhang, "Research of carrying capacity of elastic composite cylindrical roller bearing", Journal of Mechanical Transmission, vol. 37, pp. 6-9, 2013.

[9] Y. Wei, "Research on the performances of a novel roller bearingdeep cavity hollow cylindrical roller bearing", Journal of Mechanical Engineering, vol. 41, pp. 107-111, 2005.

[10] H. Xu, and C. Zhang, "Finite element analysis of roller bearing based on the plastic material models", Journal of Mechanical Engineering, vol. 46, pp. 29-35, 2010
[11] E. Armentani, R. Trapani, R. Citarella, A. Parente and M. Pirelli, "FEM-BEM numerical procedure for insertion loss assessment of an engine beauty cover", The Open Mechanical Engineering Journal, vol.7, pp. 27-34, 2013

[12] Y. Lu, Y. Zhang, Y. Yu, and L. Yu, "Nonlinear dynamics of flexible rotor system supported on fixed-tilting pad combination journal bearing", Journal of Central South University, vol. 18, pp. 610-617, 2011

[13] Z. Liu, J. Zhang, and H. Cheng, "Research on PCB collision based on ABAQUS/explicit dynamic", China Mechanical Engineering, vol. 23, pp. 1456-1461, 2012.

[14] W. Zhang, Guide to Finite Element Analysis Using ABAQUS Dynamics, ChinaTushu Publishing Limted: Hongkong, 2005.

[15] Y. Zhang, L. Xie, W. Qian, and X. Zhang, "Analysis of load distribution and stiffness characteristics of deep groove ball bearing”, Journal of Machine Design, vol. 29, pp. 45-49, 2012.

(C) Yu et al.; Licensee Bentham Open.

This is an open access article licensed under the terms of the Creative Commons Attribution Non-Commercial License (http://creativecommons.org/licenses/by-nc/3.0/) which permits unrestricted, non-commercial use, distribution and reproduction in any medium, provided the work is properly cited. 